\title{
The low cases reported in Sudan regarding a pandemic COVID-I 9 and Sudan's Health system responding
}

\begin{abstract}
Background: Novel corona virus disease (COVID-19) was reported in late 2019 in Wuhan city in China. It came in to limelight when a cluster or group of cases for pneumonia were detected and investigated with unknown causes. New cases were identified and detected in other countries, following the outbreak in China and the epidemic rapidly continued to spread globally.
\end{abstract}

Methods: According to the epidemiological view or evidence in many countries, we discuss a question often ask: is the low cases reported in Sudan regarding COVID-19 due to the less travel importation or due to current Sudan's health system in responding to the epidemic COVID-19.

Results: Our discussion can suggest and predict the cases of COVID-19 might be increase slowly regarding less travel importation with cases increase in surrounding countries, and our discussion expects the low reporting of COVID-19 cases in Sudan might be related to the level of Sudan's health systems efficiency.

Conclusion: COVID-19 proper identification and detection, and focusing on strengthening the health system with the participation of the community, are significantly important for the prevention and control of the disease not only in Sudan but in other surrounding countries and all regions.
Volume 8 Issue 4 - 2020

\author{
Abdelhakam G Tamomh,' Babiker Saad \\ Almugadam, ${ }^{2}$ Ahmed Mohammed Elnour \\ Elkhalifa ${ }^{3}$ \\ 'Department of Parasitology and Medical Entomology, Faculty \\ of Medical Laboratory Sciences, University of El Imam El Mahdi, \\ Sudan \\ ${ }^{2}$ Department of Medical Microbiology, Faculty of Medical \\ Laboratory Sciences, University of El Imam El Mahdi, Sudan \\ ${ }^{3}$ Department of Public Health, College of Health Sciences, the \\ Saudi Electronic University, Saudi Arabia
}

Correspondence: Dr.Abdelhakam G Tamomh, Department of Parasitology and Medical Entomology, Faculty of Medical Laboratory Sciences, University of El Imam El Mahdi, Kosti, Sudan,Email abdelhakam738@gmail.com; abdelhakam738@mahdi.edu.sd

Received: July 06, 2020 | Published: July 202020

\section{Introduction}

Novel corona virus disease (COVID-19) was reported in late 2019 in Wuhan city in China. It came in to limelight when a cluster or group of cases for pneumonia were detected and investigated with unknown causes. New cases were identified and detected in other countries, following the outbreak in China and the epidemic rapidly continued to spread globally. Till now, more than thirteen million and several thousand individuals have been infected with COVID-19 with several thousand mortalities. According to the epidemiological view or evidence in many countries, the estimation of the risk associated to the COVID-19 epidemic ranged from moderate risk to high. ${ }^{1-3}$ The World Health Organization (WHO) on March 11 of 2020 announced the prevalence of the novel corona virus disease is a pandemic. Currently, More than nine million confirmed cases of COVID-19 detected in 198 countries are identified and reported globally.

In Sudan, a country of forty-three million and several thousand people, a first COVID-19 case was identified on March 18, 2020, which was confirmed by a Real-Time polymerase chain reaction (PCR) test as a positive case in Khartoum a capital of Sudan. The health system surveillance and all government immediately scaled-up the efforts to contain the contagion. Samples from all suspected cases were sent to hospitals and test were performed to diagnose COVID-19 in the national reference laboratory (called Stack laboratory). Within more than a few weeks, since the first case of death reporting in Sudan, COVID-19 had appeared most of 18 states of the country, and Sudan becomes one of the countries in Africa and Eastern Mediterranean region countries which have a moderate case of COVID-19. By July 17,2020 , the number of confirmed cases detected by Sudan's surveillance system reached 10,527 cases with only 668 deaths and 5,601 recovered cases. ${ }^{5,6}$
Despite of an increased incidence of COVID-19 on a global scale, Sudan has less COVID-19 cases when compared to the other countries and its reported cases are comparable to Eastern Mediterranean region countries and some African countries. Therefore, a question usually or often asks: is low cases reported in Sudan due to less travel importation or due to the good lbad of current Sudan's health system? Are there undiscovered or miss detected cases, or perhaps a Sudanese health care system has been able to work or operate efficiently? From China, the disease spread to other countries of the world due to the level of communication. Sudan has been communicated or linked with China in form of travel, and trading, however, the grade or level of transportation between Sudan and China as well as the number of Sudanese and Chinese individuals living in the other country is not more than those observed in other countries worldwide. Thus, it can be expected that the other countries of the world are evidently infected via similar ways of communication at the same time and even early than Sudan.

In the other hand, in Africa, the world health organization (WHO) reported the first case on February 14, of 2020 in Egypt, a country close to Sudan, which it's one of the highest importation countries risk reported in Africa followed by Algeria and South Africa. ${ }^{3,5,7}$

Sudan has been communicated with all border African countries, especially Egypt which is in the north border of Sudan, and the level of transportation is more when compared to Sudan and China in the form of trade, tourist, and traveling. Hence, it can be predicted that the cases of COVID-19 will increase in Egypt if the transportation continues between the two countries. A low reporting of COVID-19 cases in Sudan might be attributed to less detected cases or due to the transparency in cases reported, less importation of individuals from infected countries, and may relate to the significant chance that 
can affect the transmission rate and cause the lower discovery of the epidemic disease in a specific area or region. Regarding transparency, Sudan has evidently tried to meet and adhere to the standard and involvements.

It is better to evaluate Sudan's health system infrastructure, to clarify the sensitivity of Sudan's health system toward the outbreak of COVID-19. The economic challenges faced by the government leads to deteriorating of living conditions. Sudan has gathered all it is efforts, facilities, and materials for detection, awareness, and control of the disease.

Sudan's health system contains its private and governmental medical universities, care centers, and general and private medical laboratories, throughout the country. Federal ministry of health with the cooperation of Stack reference and national laboratory is responsible directly for laboratory investigation or diagnosis of the COVID-19. Currently, a few other private laboratories in Khartoum state in Sudan are providing and giving diagnostic services in the country. Medical care services for COVID-19 cases are provided by most of the hospitals. Sudan's primary health care system is responsible for providing primary health care for the remote areas of Sudan. In addition, health volunteers and teams of volunteers formed and prepared by the private and the government in order to understand the situation and quick responses to the disease have increased the benefits of public or general authorities.

More than forty medical colleges affiliated to more than thirtyone governmental universities in collaboration with more than ten private medical colleges in Sudan has trained thousands of medical doctors, nurses, clinical specialists, and basic medical sciences, who significantly participated and contributed in the health education, explaining the risk of the COVID-19 with the preparation of medical laboratories and clinical centers in responding to the disease. For the epidemiology community of Sudan, few hundreds of trained workers prepared from epidemiologists in all states, for epidemic consultations and investigative services as a part of the federal ministry of health distributed in all regions of Sudan for better control of the epidemic disease, despite the economic deterioration challenges. Sudan's federal ministry of health has taken more fundamental processes towards communicable diseases, such as polio, malaria, and tuberculosis. ${ }^{8-10}$ Many regions or areas in Sudan still need quick improvements, despite the significant achievements of the country in responding to COVID-19. The most and major challenges are cross-sectoral contribution and collaboration. Following a month after the first case of COVID-19 confirmed in Sudan, the federal ministry of health has taken efforts, by improving the participation and the role of people in social distancing and voluntary house isolation. Accordingly, these measures will provide a harmonized or good response and prevent the country from the rapid distribution of the epidemic disease. Other challenges of Sudan's health system are dealing with COVID-19 outbreak was data managing and presenting or introducing a definition for a sensitive case of the disease to identify or detect more cases. In spite cases on a daily basis in Sudan's, the country tries to maintain transparency of the report, and the accuracy for the detection of the cases reporting in all Sudan states. Therefore, inconsistencies for statistical data that reported, sometimes indicators or indexes cannot be ascertained and quantified precisely. Despite of COVID-19 epidemic in Sudan, the country's health system has attempted to detect the cases on a daily basis. However, I could be said that Sudan has low cases of COVID-19 compared to other countries with similar economic and development status. Sudan has low cases when compared with other countries in the Eastern Meditation region except for countries facing war, such as Syria, Yemen, and Libya. Only Syria with 338 cases reported recently. ${ }^{5}$ Due to improper of socio-economic factors, lack of acceptable health system, including enough number of medical care centers, medical and clinical laboratory facilities, and sensitive health surveillance systems on these countries, it has managed to cope up with the situation in better manner when compared to its neighboring counterparts.

In any country, improved case identification and detection are significantly important for the control of the epidemic. Also, other countries will be affected in a short time of period, if there are a lackcontrolled epidemic and undetected cases in the country. ${ }^{11,12}$

In conclusion, COVID-19 proper identification and detection are significantly important for the prevention and control of the disease not only in Sudan but in other surrounding countries and all regions. Similarities in environmental and geographical factors, habitats, lifestyle, and cultures between the countries of the same region, might affect individuals' or populations' health and behaviors, thus affect the infrastructures of the economy. Therefore, focusing on strengthening the diagnostic ability and capacity of these countries with the participation of the community, and taking the lessons learned from similar or near socio-economic countries affected in the region with the disease.

\section{Acknowledgments}

None.

\section{Conflicts of interest}

Authors declare that there is no conflict of interest.

\section{References}

1. Zhu N, Zhang D, Wang $\mathrm{W}$, et al. A novel coronavirus from patients with pneumonia in China, 2019. N Engl J Med. 2020;382(8):727-733.

2. Boldog P, Tekeli T, Vizi Z, et al. Risk assessment of novel coronavirus COVID-19 outbreaks outside China. J Clin Med. 2020;9(2):571.

3. Gilbert M, Pullano G, Pinotti F, et al. Preparedness and vulnerability of African countries against importations of COVID-19: a modelling study. Lancet. 2020;395(10227):871-877.

4. Read JM, Bridgen JR, Cummings DA, e al. Novel coronavirus 2019$n C o V$ : early estimation of epidemiological parameters and epidemic predictions. medRxiv; 2020. $11 \mathrm{p}$.

5. Daily report on COVID-19 in the Eastern Mediterranean Region. WHO Regional Office for the Eastern Mediterranean; 2020.

6. Federal Ministry of Health, The Republic of Sudan, corona in Sudan daily report; 2020.

7. Nkengasong JN, Mankoula $\mathrm{W}$. Looming threat of COVID-19 infection in Africa: act collectively, and fast. Lancet. 2020;395(10227):841-842.

8. Sahal N, Reintjes R, Eltayeb EM, et al. Assessment of core activities and supportive functions for the communicable diseases surveillance system in Khartoum state, Sudan, 2005-2007. East Mediterr Health J. 2012;16(12):1204-1210.

9. Elnour FA, Alagib MEA, Bansal D, et al. Severe malaria management: current situation, challenges and lessons learned from Gezira State, Sudan. Malar J. 2019;18(1):170. 
10. Elduma AH, Mansournia MA, Foroushani AR, et al. Assessment of the risk factors associated with multidrug-resistant tuberculosis in Sudan: a case-control study. Epidemiol Health. 2019;41:e2019014.

11. Fisher D, Heymann D. Q\&A: The novel coronavirus outbreak causing COVID-19. BMC Medicine. 2020;18:57.
12. Li JY, You Z, Wang Q, et al. The epidemic of 2019-novel coronavirus (2019-nCoV) pneumonia and insights for emerging infectious diseases in the future. Microbes Infect. 2020;22(2):80-85. 